\title{
Reflexiones acerca de la brecha digital en la educación a distancia durante la pandemia de COVID-19
}

\author{
María Belén Vertiz Manco \\ maria.vertiz@unmsm.edu.pe \\ Universidad Nacional Mayor de San Marcos (Lima, Perú) \\ (iD) https://orcid.org/0000-0002-2349-2197
}

\section{Claudia Gisell Salas Oscco}

claudia.salas@unmsm.edu.pe

Universidad Nacional Mayor de San Marcos (Lima, Perú)

(iD https://orcid.org/0000-0002-9750-4373

\begin{abstract}
Resumen
Dentro del contexto generado por un acontecimiento de efecto global como lo es la actual pandemia de COVID-19, se puede aseverar que -junto con el adecuado aprovisionamiento de las necesidades básicas para la supervivencia humana- la educación es uno de los factores de la cotidianidad a los que se les dedica mayor concentración por preservar. En ese sentido, puede contarse con una considerable ventaja: la existencia de una metodología alternativa a la coexistencia de maestros y alumnos en un espacio físico común, que, si bien no constituía una opción de aplicación generalizada, tampoco resultaba completamente ajena al estudiante promedio en un país como el Perú. Nos referimos a la educación a distancia. Sin embargo, mientras que los beneficios inmediatos de adoptar este sistema, a primera vista tan accesible, de hecho, son muchos, la imposición intempestiva de la educación a distancia en todos los niveles educativos en el país no se instauró sin retos ni reveses. La ampliación de la brecha digital -antes una realidad relativamente pasiva de ignorar para aquellos que no se percibían como especialmente afectados por esta- alcanzó nuevas alturas con una velocidad vertiginosa, y se convirtió en una preocupación de suma relevancia debido a su efecto sobre la habilidad de los estudiantes peruanos de continuar recibiendo su instrucción bajo la modalidad virtual o en su defecto, por visibilizar de manera contundente los efectos de la cada vez más aguda desigualdad social que perjudica al país.
\end{abstract}

\section{Palabras clave}

Brecha digital, educación, educación a distancia, TIC, pandemia

\section{(c) (1)}




\title{
Reflections on the digital gap in long-distance education during the COVID-19 pandemic
}

\author{
María Belén Vertiz Manco \\ maria.vertiz@unmsm.edu.pe \\ Universidad Nacional Mayor de San Marcos (Lima, Perú) \\ (iD) https://orcid.org/0000-0002-2349-2197
}

Claudia Gisell Salas Oscco

claudia.salas@unmsm.edu.pe

Universidad Nacional Mayor de San Marcos (Lima, Perú)

iD https://orcid.org/0000-0002-9750-4373

\begin{abstract}
Within the context generated by an event with a global effect such as the current COVID-19 pandemic, it can be asserted that -along with the adequate provision of basic needs for human survival- education is one of the factors of daily life to which the greatest concentration for preserving is dedicated. In this sense, there is a considerable advantage: an alternative methodology to the coexistence of teachers and students in a common physical space, which, although it was not an option for general application, was not completely alien to the average student in a country like Peru. We refer to long-distance education. However, while the immediate benefits of adopting this system, at first glance so accessible, are in fact many, the untimely imposition of long-distance education at all educational levels in the country was not established without challenges or setbacks. The widening of the digital gap -once a relatively passive reality to ignore for those who did not perceive themselves as particularly affected by it- reached new heights with breakneck speed and became a major concern due to its effect on the ability of Peruvian students to continue receiving their instruction under the virtual modality, or, failing that, to make visible in a forceful way the effects of the increasingly acute social inequality that harms the country.
\end{abstract}

\section{Keywords}

Digital gap, education, long-distance education, ICT, pandemic 


\section{Introducción}

La educación no es un proceso que pueda impartirse de manera estática: a medida que los contextos socioculturales van variando con el tiempo, la educación -como necesidad primordial- se adapta a ellos. Con el transcurrir de los ańos, hemos visto a las formas de enseñanza, los enfoques y los paradigmas de la educación atravesar incontables cambios, ya fuesen menores o mayores, trascendentales, o incluso redundantes. Es en el marco de aquella eterna carrera por amoldarse a las necesidades de los estudiantes de "hoy" que -una vez que los recursos tecnológicos e informáticos adecuados para tal propósito se masificaron- finalmente se logró desarrollar un sistema que cubriera las necesidades de aquellos que anhelan recibir una educación, pero que no se ven en las posibilidades de ubicarse en el tradicional espacio físico destinado a esto: la educación a distancia.

En el caso del Perú, Rodríguez (2013) señala que podemos hallar el antecedente más antiguo en materia de educación a distancia en una experiencia realizada por la Pontificia Universidad Católica del Perú alrededor de los años 50. Sin embargo, no fue sino hasta más de medio siglo más tarde, en 2007, que esta modalidad de estudios fue aceptada con la firme resolución de promover su desarrollo para lograr su acreditación.

Dentro del contexto de la pandemia de COVID-19 que atravesamos actualmente, la educación a distancia ha hallado tanto su oportunidad de brillar como su mayor desafío. A medida que el veloz e inexorable avance de la enfermedad se volvía innegable -incluso para los más escépticos-, todos los países alrededor del mundo fueron adoptando diversas medidas para proteger las vidas y la salud del público en general, medidas que, si bien en realidad no pueden ser contabilizadas como homogéneas, comparten un significativo denominador común: la cancelación de las clases presenciales en todos los niveles educativos.

La educación, como pilar de la sociedad, no podía ser relegada a la categoría de preocupación secundaria -incluso dentro de un panorama en que la preservación de la salud de las personas, así como un adecuado acceso al abastecimiento de alimentos y agua era primordial-, por lo que países enteros se volvieron hacia el único medio que les podría permitir mantener un ritmo relativamente consistente en la educación de niños y jóvenes.

En los casos de una amplia mayoría de países desarrollados, en los cuales esta metodología ya había sido introducida en una escala significativa y no resultaba una mayor innovación, instaurar la educación a distancia fue una respuesta inmediata que gozó de una acogida positiva. En contraposición, en aquellos países en los que la educación a distancia no había sido aún probada en la magnitud en la que intempestivamente se veía siendo aplicada, las reacciones variaron, y fue el alivio ante la continuidad de las clases la respuesta más optimista, diametralmente opuesta a la angustia surgida entre las cantidades masivas de familias que veían la educación de los niños y jóvenes truncarse ante la infranqueable brecha digital.

\section{Discusión}

La investigación antropológica sobre la educación a distancia toma como referente la antropología personalista basada en el pensamiento filosófico de Wojtyla, el cual ubica a 
la persona como el centro, incorporando cuerpo y alma en un proceso integrador en el que hombre es determinado por sus acciones, las cuales, a su vez, son autorreferenciales. La antropología personalista es planteada bajo esta perspectiva que analiza, entre varios otros temas, el aspecto del acompañamiento en la educación, específicamente postulando que la educación se basa en acompañar a una persona durante este proceso de aprendizaje. La tutoría es uno de los puntos más importantes en la educación a distancia, ya que, el acompañamiento es un aprendizaje mutuo, motiva al individuo a descubrir su vocación mientras contribuye a su reforzamiento en el docente (Gonzalez Iglesias \& De la Calle Maldonado, 2020).

Sin embargo, cabe destacar que, hasta antes de la pandemia, las investigaciones antropológicas de la educación a distancia eran más bien escasas, lo que generaba un vacío teórico dentro de la disciplina y que resultaba extraño al tratarse de una metodología educativa cuya concepción fue revolucionaria en su momento. Mientras tanto, desde la aparición del coronavirus, la atención volcada a la temática desde todas las ciencias sociales se ha despuntado, y cabe mencionar -entre otras- las contribuciones de García Aretio (2020), Díaz Barriga (2020), Plá (2020) y Díaz Villalobos (2020), que circulaban alrededor de las nuevas terminologías introducidas en materia de educación a distancia así como los desafíos y limitaciones de esta, y la postura de los docentes al afrontar un cambio de paradigma tan profundo como inesperado.

Dentro de las consideraciones más urgentes, los desafíos y limitaciones de la educación a distancia en el contexto de la pandemia pueden resumirse de manera particularmente adusta en la desmedida expansión y visibilización de la brecha digital.

En el caso del Perú, un país con una inmensa mayoría de ciudadanos y residentes dedicados a las actividades informales como la principal fuente de sustento de familias enteras, la precariedad económica de por sí existente se dejó sentir de manera brutal entre los estratos cuyos ingresos dependían únicamente de los sectores determinados como no esenciales. Situándonos en el contexto nacional, la expresión de la desigualdad en la pobreza, según Delgado Pugley (2020) -basándose en los datos de OXFAM (Oxford Committee for Famine Relief)-, el 20,5\% del país se encuentra en situación de pobreza, mientras que el $40,1 \%$ de la población forman parte de la clase media, el $25 \%$ son empleados del sector formal y el 75\% dependen de ingresos inestables.

Lloyd (2020) refiere que el término "brecha digital" surge en Estados Unidos alrededor de la década de 1990 para referirse a la desigualdad en el acceso de las TIC; sin embargo, también se incluyen en esta definición las capacidades digitales y los valores que se asocian al uso eficiente de las TIC. Por su lado, Carbajal (2013) enfatiza la relevancia de los valores asociados a las TIC en el contexto de la educación a distancia, por lo que es innegable que tanto la capacidad de cambio, de adaptación y una actitud de asertiva frente a tales modificaciones son aptitudes sumamente valiosas ante la eterna evolución de la tecnología, por lo que la habilidad de los profesionales de mantenerse a la vanguardia de un entorno virtual de aprendizaje (EVA) será clave en su papel de guías para los estudiantes.

En esta "nueva normalidad" en la que nos ha enmarcado la pandemia, la presencia de estas brechas se ve evidenciada por las condiciones propias de la educación a distancia, las 
cuales pueden ocasionar el fracaso de la aplicación de contenidos pedagógicos a raíz de una carencia de recursos y/o de habilidades en el manejo de las TIC.

Osio Havriluk (2010) señala el cambio total que se ha venido desarrollando dentro de la sociedad a pesar de los nuevos retos que impone la digitalización, dado que el uso de las TIC permite la creación de nuevas estructuras que ejercen influencia sobre prácticamente todos los aspectos de nuestra cotidianidad, lo que a su vez, había acelerado la visibilización de la brecha digital que distinguía a pobres de ricos acorde con el acceso que tienen a los mismos recursos digitales.

Las iniciativas relativas a las TIC y a la modernización de la educación en el Perú han sido numerosas, pero los profundos problemas de infraestructura y la falta de servicios básicos que pueden hallarse desde la comunidad más aislada hasta las ciudades más metropolitanas nulificaban cualquier avance sustancial que pudiese realizarse y ocasionalmente se veían incluso asistidos en tal fiasco por la aplicación de medidas poco atinadas, como, por ejemplo, mesurar que la recepción de una laptop se sobrepondría a la falta de servicios confiables de electricidad e internet en el área (Quintanilla Cóndor, et al. 2019).

El contexto de la pandemia genera una terrible proclividad a discriminar automáticamente a aquellos estudiantes cuyas circunstancias los privan de acceso a recursos para acceder a las TIC, así como a los docentes que carecen de los conocimientos necesarios para abordar esta modalidad en una frecuencia absoluta.

Lloyd (2020) apunta la manera en que la desigualdad expresada a través de la brecha digital afecta indisputablemente la productividad y el rendimiento académicos: un estudiante que carece de las facilidades para plegarse a esta metodología se verá lógicamente limitado en el momento de emprender sus actividades académicas, mientras que la situación de los docentes se ve ligeramente más esperanzadora dada su facilidad comparativa para acceder a los conocimientos necesarios para adquirir habilidades en las TIC. Ahora bien, de acuerdo con Toledo, et al. (2020), la gestión de la educación a distancia continúa siendo terreno inexplorado, por lo que no existe una serie de criterios propios para la realización de esta.

\section{Conclusiones}

La brecha digital, el principal desafío de la educación a distancia, consiste en nada más y nada menos que la expresión tecnológica de la pobreza, presente en todo el mundo y uno de los problemas vigentes más apremiantes del contexto sociocultural de nuestra época.

Mientras que la pandemia de COVID-19 - una circunstancia sumamente irregularelevó el despunte de la brecha digital a una amenaza inminente hacia la continuidad del proceso educativo, esta ya venía ejerciendo su influencia sobre miles de hogares dependientes de ingresos erráticos a lo largo de décadas, y únicamente ahora se le otorga tal nivel de atención debido a su repentina relevancia respecto de la capacidad de un alumno de continuar recibiendo clases bajo la modalidad de educación a distancia o si, por el contrario, este estudiante deberá pasar a formar parte de las filas de aquellos que se deslizan a través de las grietas de una metodología que se inclina en favor de aquellos que cuentan con la buena fortuna de hallarse del lado más auspicioso de la desigualdad social. 
En este contexto, podemos concluir que la aplicación efectiva y eficiente del objetivo básico de la educación a distancia -impedir una interrupción permanente de las clases o su cancelación oficial durante 2020 - fue cumplido. Sin embargo, tal éxito no vendría a ser sino una victoria pírrica en el rostro de los incontables estudiantes afectados por sus poco favorables circunstancias que los privan de amoldarse al nuevo paradigma educativo, así como de los docentes que no logran adaptarse a la nueva realidad de su profesión y a quienes la pérdida del acompañamiento en el espacio físico compartido en adición al empleo de las TIC ha logrado desestabilizar como educadores.

\section{Referencias bibliográficas}

Carbajal, G. (2013). Competencias profesionales del docente en un entorno virtual de aprendizaje. En J. Domínguez Granda y C. Rama (Eds.), La educación a distancia en el Perú (pp. 251-289). Universidad Católica Los Ángeles. Obtenido de https:// virtualeduca.org/documentos/observatorio/la_educacion_a_distancia_en_peru.pdf

Delgado Pugley, D. (2020). La covid-19 en el Perú: una pequeña tecnocracia enfrentándose a las consecuencias de la desigualdad. Análisis Carolina, 1-16. https:// doi.org/10.33960/AC_26.2020

Díaz Barriga, Á. (2020). La escuela ausente, la necesidad de replantear su significado. En H. Casanova Cardiel (Coord.), Educación y pandemia: una visión académica (pp. 1929). Ciudad de México: Universidad Nacional Autónoma de México, Instituto de Investigaciones sobre la Universidad y la Educación. Obtenido de https://www.iisue. unam.mx/investigacion/textos/educacion_pandemia.pdf

Díaz Villabolos, M. S. (2020). Programa pedagógico con utilización del aula virtual basado en la pedagogía constructivista para el logro de las capacidades de ciencia tecnología y ambiente en la química orgánica [Tesis de maestría]. Lambayeque, Perú: Universidad Nacional Pedro Ruiz Gallo. Obtenido de https://repositorio.unprg.edu.pe/ bitstream/handle/20.500.12893/8134/BC-4567\%20DIAZ\%20VILLALOBOS. pdf?sequence $=1$ \&isAllowed $=y$

García Aretio, L. (2020). Bosque semántico: ¿educación/enseñanza/aprendizaje a distancia, virtual, en línea, digital, eLearning...? Revista Iberoamericana de Educación a Distancia, 23(1), 9-28.

http://dx.doi.org/10.5944/ried.23.1.25495

Gonzalez Iglesias, S. M., y De la Calle Maldonado, C. (2020). El acompañamiento educativo, una mirada ampliada desde la antropología personalista. Scientia et Fides, 8(1), 183-203. Obtenido de https://apcz.umk.pl/czasopisma/index.php/SetF/article/ view/SetF.2020.012

Lloyd, M. (2020). Desigualdades educativas y la brecha digital en tiempos de COVID-19. En UNAM, Educación y Pandemia (pp. 115-122). México: UNAM. Obtenido de https://www.iisue.unam.mx/investigacion/textos/educacion_pandemia.pdf

Osio Havriluk, L. (2010). El teletrabajo: una opción en la era digital. Observatorio Laboral Revista Venezolana, 3(5), 92-109. Obtenido de https://www.redalyc.org/ pdf/2190/219014912006.pdf 
Plá, S. (2020). La pandemia en la escuela: entre la opresión y la esperanza. En UNAM, Educación y pandemia (pp. 30-39). México: UNAM. Obtenido de https://www.iisue. unam.mx/investigacion/textos/educacion_pandemia.pdf

Quintanilla Cóndor, C. N., Oré Rojas, J. J., \& Quispe Ccora, C. R. (2019). Análisis del programa de una computadora por niño en instituciones educativas en zonas de exclusión y pobreza: caso Perú. Revista Iberoamericana De Educación, 79(1), 71-95. Obtenido de https://doi.org/10.35362/rie7913391

Rodríguez, J. (2013). Aproximaciones a la educación a distancia en el Perú. En J. Domínguez Granda y C. Rama (Eds.), La educación a distancia en el Perú (pp. 37-53). Universidad Católica Los Ángeles. Obtenido de https:/virtualeduca.org/ documentos/observatorio/la_educacion_a_distancia_en_peru.pdf

Toledo, M., Correa, S., Valdés, M., \& Hadweh, M. (2020). Modelo de gestión educativa para programas en modalidad virtual de aprendizaje. Revista de Ciencias Sociales, 16(2), 286-298. Obtenido de https://www.researchgate.net/publication/343774518_Modelo_de_gestion_educativa_para_programas_en_modalidad_virtual_de_aprendizaje 